\title{
Value-Based Resource Management in High-Performance Computing Systems
} Dylan Machovec ${ }^{1}$, Cihan Tunc ${ }^{3}$, Nirmal Kumbhare ${ }^{3}$, Bhavesh Khemka ${ }^{1}$, Ali Akoglu ${ }^{3}$, Salim Hariri ${ }^{3}$, Howard Jay Siegel ${ }^{1,2}$

\author{
${ }^{1}$ Department of Electrical and Computer Engineering \\ ${ }^{2}$ Department of Computer Science \\ Colorado State University, Fort Collins, CO 80523, USA \\ DJMachov@rams.colostate.edu, bhavesh.khemka@gmail.com, HJ@colostate.edu
}

${ }^{3}$ Department of Electrical and Computer Engineering

University of Arizona, Tucson, AZ 85721, USA

CihanTunc@email.arizona.edu, nirmalk@email.arizona.edu, akoglu@ece.arizona.edu, hariri@ece.arizona.edu

\begin{abstract}
We introduce a new metric, Value of Service (VoS), which enables resource management techniques for high-performance computing (HPC) systems to take into consideration the value of completion time of a task and the value of energy used to compute that task at a given instant of time. These value functions have a soft-threshold, where the value function begins to decrease from its maximum value, and a hard-threshold, where the value function goes to zero. Each task has an associated importance factor to express the relative significance among tasks. We define the value of a task as the weighted sum of its value of performance and value of energy, multiplied by its importance factor. We also consider the variation in value for completing a task at different time; the value of energy reduction can change significantly between peak and non-peak periods. We define VoS for a given workload to be sum of the values for all tasks that are executed during a given period of time. Our system model is based on virtual machines (VMs), where each dynamically arriving task will be assigned to a VM with a resource configuration based on number of homogenous cores and amount of memory. Based on VoS, we design, evaluate, and compare different resource management heuristics. This comparison is done over various simulation scenarios and example experiments on an IBM blade server based system.
\end{abstract}

\section{CCS Concepts}

- Social and professional topics $\sim$ System management - Computer systems organization Cloud computing - Software and its engineering $\sim$ Real-time schedulability - Software and its engineering $\sim$ Scheduling

Permission to make digital or hard copies of all or part of this work for personal or classroom use is granted without fee provided that copies are not made or distributed for profit or commercial advantage and that copies bear this notice and the full citation on the first page. Copyrights for components of this work owned by others than ACM must be honored. Abstracting with credit is permitted. To copy otherwise, or republish, to post on servers or to redistribute to lists, requires prior specific permission and/or a fee. Request permissions from Permissions@acm.org.

ScienceCloud'16, June 01 2016, Kyoto, Japan

(C) 2016 ACM. ISBN 978-1-4503-4353-4/16/06 ...\$15.00

DOI: http://dx.doi.org/10.1145/2913712.2913716

\section{Keywords}

energy-aware resource allocation; modeling; performance metrics; resource management; Value of Service.

\section{INTRODUCTION}

In today's high-performance computing $(H P C)$ systems, there is a critical need to provide services in a timely manner that meets user performance requirements, while also being energy efficient. For example, in exascale computing, the power goal is to be under $20 \mathrm{MW}$ power and that requirement cannot be met with current resource management techniques [23]. As a result, considering the complexity and diversity of HPC applications and systems, there are strong interests in development of new resource management strategies that are both energy effective and scalable.

In this paper, we introduce a new metric, Value of Service $(\operatorname{VoS})$ that enables resource management algorithms to consider the current time (e.g., peak vs. non-peak period) in evaluating the value of completion time of a task and the value of energy used to compute a task at a given instant of time. For example, in the context of weather prediction, the value of storm prediction task is relative to storm occurrence time. The value of providing the prediction in 24 hours prior to occurrence is more valuable than making the same prediction only 2 hours. And, the importance of a storm prediction task, due to its life criticality, is higher than that associated with other tasks (e.g., predicting weather temperature). Thus, for resource allocation, our value-based scheduling model uses value metric to prioritize a task responsible for storm forecasting over a task responsible for a temperature forecast.

Our measure of the effectiveness of the resource management algorithms is based on $\operatorname{VoS}$. VoS for a given workload assignment can be computed as the collective value of workload tasks for a given period of time. In this work, value at a given period of time consists of two components: (1) value of performance, based on task completion time, and (2) value of energy, based on the energy used to execute the task. Each task type has an associated monotonically decreasing performance value function and energy value function. Value functions are designed by the user submitting the task in collaboration with the system owner. These value functions have a soft-threshold, where the value function begins to decrease from its maximum value, and a hard-threshold, where the value function goes to zero. The performance and 
energy value components are given relative weights. In addition, each task has an associated importance factor, to express the relative significance among tasks. We define the value of a task at a given time as the weighted sum of its value of performance and value of energy, multiplied by the importance factor of that task. In addition, if either the value of performance or value of energy is zero, then the value of the task is zero. We define $V o S$ to be sum of the values for all executed tasks during a given period of time. Based on $V o S$, we design, evaluate, and compare different resource management heuristics both by simulation and by experimentation on an IBM blade server.

Our system model is based on virtual machines $(V M s)$ (and the same concept can be applied to containers as well), where each task is assigned to a VM with a resource configuration based on number of homogenous cores and amount of memory. Using historical and experimental information, for each task type, we obtain the task type's estimated execution time and energy consumption. We consider an environment with dynamically arriving tasks assigned to VMs. Also, we assume that the system is oversubscribed such that not all tasks can begin execution immediately upon arrival.

Our contributions include:

- Formulation and use of value-based metrics for resource management for a given period of time.

- Design, analysis, and comparison of resource management heuristics using both simulation and experiments on an actual system.

- Derivation of a data-driven model methodology to represent task behavior and to predict their energy consumption.

The rest of the paper is organized as follows. We give an overview of our system model and environment in Section 2. The $V o S$ metric is introduced in Section 3, followed by our resource management heuristics in Section 4. We present our simulation evaluations in Section 5. In Section 6, we describe our data-driven modeling and provide experiment results. Related work is discussed in Section 7, followed by our conclusions in Section 8.

\section{SYSTEM MODEL}

We assume that the HPC system consists of homogenous clusters. Tasks arrive dynamically with user-specified soft and hard completion deadlines. This constraint is coupled with an energy consumption constraint imposed by the service provider. The service provider applies these constraints using a value function representing the value earned.

Each task is executed on an individual VM with the specified resource configuration as the number of cores and the amount of memory. Each task belongs to some task type which consists of tasks with similar computational characteristics. For each task type, there is a set of allowable resource configurations to achieve non-zero value if their execution could start immediately and a known estimated task execution time and estimated task energy usage, determined by historical data or experimentation. It is common in the resource management literature to assume the availability of this information, e.g., [14] and [26].

\section{VALUE OF SERVICE FOR RESOURCE MANAGEMENT}

There is a large body of work on resource management strategies for HPC systems targeting various metrics such as system resource utilization, task execution time, and total power/energy consumption. Utility functions ([12], [13], [17], [25]) have been shown to be an effective performance measure for resource management in an oversubscribed environment. Our work builds on this previous research to combine performance and energy to define VoS.

The value for a task is a monotonically-decreasing function of an objective (e.g., completion time or energy) that specifies the value earned by completing a task at a given instant of time. We assume that the task completion value depends on the time of completing the task execution. The value of energy reduction at peak period of energy consumption is significantly larger than the value of energy reduction when the energy consumption is very low. The shape of a particular value function for a given task depends on a set of parameters determined by the end-user in conjunction with the service provider. These parameters are illustrated in Figure 1. The soft threshold specifies the limit on an objective until which the value earned by task is maximum. Beyond soft threshold, the value starts decreasing until the objective reaches the hard threshold. The hard threshold specifies a limit on a given objective, beyond which zero value is earned.

We model two value functions in our studies: (a) completion time (performance) value function and (b) energy value function. The performance value function for a task depends on the completion time. The user defines the soft and hard thresholds for the task completion time. The soft threshold specifies the limit on task completion time until which the performance value earned by task is maximum. Beyond soft threshold, the performance value starts decreasing until the task completion time reaches the hard threshold. The hard threshold specifies a limit on completion time, beyond which zero value is earned. Similarly, the energy value function of a task depends on the energy consumption for its execution at a given period of time. The soft and hard energy threshold value functions can be defined by the service provider.

We model the $V o S$ based on performance and energy values. A contribution of our model is that we consider a weighted sum of both performance and energy metrics for $V o S$ based resource management decisions at a given period of time, further refined by a relative importance factor among tasks.

In our study, we are using linear decay in value in between the soft threshold and the hard threshold of an objective. A value function is formulated as follows:

- The value $\left(v\left(\operatorname{Tas}_{i}, t\right)\right)$ represents the value for completing task $i$ at an instant $t$ with respect to a given objective (e.g., execution time or energy consumption).

- The max value $\left(v_{\max }\left(\operatorname{Tas}_{i}\right)\right)$ represents the maximum value that can be achieved by completing task $i$ at any point in time.. Note that this max value will change depending on the period at which the resource management is performed.

- The soft threshold $\left(T h_{\text {Soft }}\left(\operatorname{Task}_{i}\right)\right)$ defines the limit on the objective value for task $i$ to gain the maximum possible value $\left(v_{\max }\left(\operatorname{Task}_{i}\right)\right)$.

- The hard threshold $\left(\operatorname{Th}_{\text {Hard }}\left(\operatorname{Tas}_{i}\right)\right)$ defines the final acceptable limit on the objective value for which task $i$ 's completion can gain any non-zero value.

- The min value $\left(v_{\min }\left(\operatorname{Task}_{i}\right)\right)$ represents the minimum positive value that can be achieved by completing the task $i$.

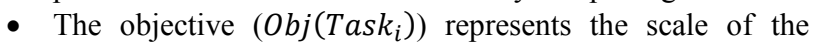
parameter (can be execution time, energy, resiliency, etc.) corresponding to which the value of the function is measured for task $i$ for a given instant of time or period. 
If task $i$ 's execution does not complete before $T h_{H a r d}\left(t_{i}\right)$, the value drops to zero. In between $T h_{\text {Soft }}\left(t_{i}\right)$ and $T h_{\text {Hard }}\left(t_{i}\right)$, the value for task $i$ decreases linearly from $v_{\max }\left(t_{i}\right)$ to $v_{\text {min }}\left(t_{i}\right)$. In this study, we assume this function to be linear, however, other functions can be also considered in a similar way.

In Figure 1, a fixed value is gained till the soft threshold $\left(\operatorname{Th}_{\text {Soft }}\left(t \operatorname{Task}_{i}, t\right)\right)$, which is defined by $v_{\max }\left(\operatorname{Task}_{i}, t\right)$ (Equation 1(a)), and no value is gained after the hard threshold

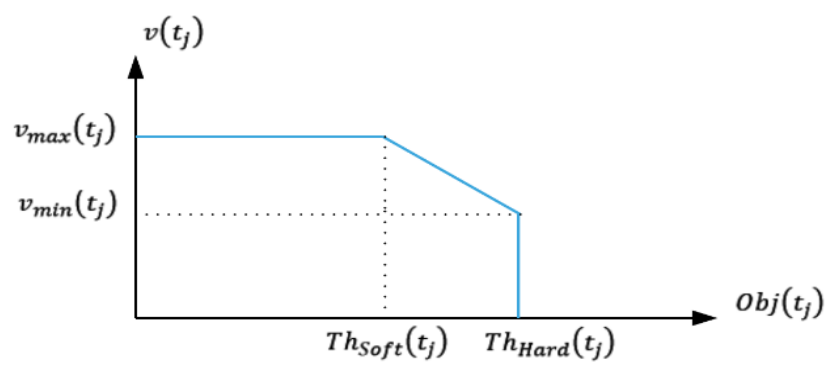

Figure 1. General formulation for value vs objective and threshold values.

$\left(T h_{\text {Hard }}\left(t_{i}\right)\right)$ (Equation 1(c)). In between, the rate of reduction in the value is captured by Equation 1(b). The difference between the current state of the objective and its hard threshold determines the magnitude of the value gain, which is captured by $\left(\operatorname{Obj}\left(t_{i}\right)-\right.$ $\left.T h_{\text {Hard }}\left(t_{i}\right)\right)$. Here, we note that for the performance and energy value functions, task completion time (seconds) and energy consumption (Joules) are the metrics used.

Task value $\left(V\left(\right.\right.$ Task $\left.\left._{i}, t\right)\right)$, the total value earned by completing task $i$ at a given period $t$, is the weighted sum of earned performance and energy values, as shown in Equation 2. The

a) if $\left(0 \leq \operatorname{Obj}\left(\operatorname{Task}_{i}, t\right) \leq T h_{\text {Soft }}\left(\operatorname{Task}_{i}, t\right)\right)$ then

$$
v\left(\operatorname{Task}_{i}, t\right)=v_{\max }\left(\operatorname{Task}_{i}, t\right)
$$

b) else if $\left(\operatorname{Th}_{\text {Hard }}\left(\operatorname{Task}_{i}, t\right)>\operatorname{Obj}\left(\operatorname{Task}_{i}, t\right)\right.$

$$
\begin{aligned}
& \left.>T h_{\text {Soft }}\left(\operatorname{tTask}_{i}, t\right)\right) \text { then } \\
& \left.v\left(\operatorname{Task}_{i}, t\right)=\left(\operatorname{Obj}_{\left(\operatorname{Task}_{i},\right.}\right)-\operatorname{Th}_{\text {Hard }}\left(t_{i}\right)\right) \\
& * \frac{v_{\text {max }}\left(t_{i}\right)-v_{\text {min }}\left(t_{i}\right)}{T h_{\text {Soft }}\left(t_{i}\right)-T h_{\text {Hard }}\left(t_{i}\right)}+v_{\text {min }}\left(t_{i}\right),
\end{aligned}
$$

c) else if $\left(\operatorname{Obj}\left(t_{i}\right) \geq T h_{\text {Hard }}\left(t_{i}\right)\right)$ then,

$$
v\left(t_{i}\right)=0 \text {. }
$$

$w_{p}$ and $w_{e}$ coefficients are then used for adjusting the weight given to performance and energy values. The importance factor $\gamma^{t_{i}}$ expresses the relative importance among tasks. In addition, if either the value of performance or value of energy is zero, then the total value of the task is zero.

$V\left(\operatorname{Task}_{i}, t\right)=\gamma^{\left(t_{i}\right)}\left(w_{p} x v_{p}\left(\right.\right.$ Task $\left._{i}, t\right)+w_{e} * v_{e}\left(\right.$ tTask $\left.\left._{i}, t\right)\right)$

$\operatorname{VoS}$ (Equation 3 ) is the total value gained by $n$ tasks in the workload that are completed for a given time interval

$$
\operatorname{VoS}(t)=\sum_{i=1}^{n} V\left(\text { Task }_{i}, t\right)
$$

\section{RESOURCE MANAGEMENT HEURISTICS}

A mapping event is when resource management decisions are made. In our environment, mapping events occur every minute, as typical at facilities such as those used by DoD [17]. At a mapping event, all tasks that have arrived in the system and have not started execution and have not been dropped are considered mappable since the last mapping event. Our heuristics use the concept of place-holders as temporary reservations, which we introduced in our earlier work [13]. They resulted in better resource allocations compared to permanent reservations for dynamically arriving tasks with a monotonically decreasing performance measure (e.g., [1], [15]). Making reservations helps to prevent the starvation of tasks that need large amount of resources. During a mapping event, place-holders provide the benefits of reservations to prevent the assignment of lower-value tasks that may disturb the execution of higher-value tasks needing a larger set of resources. At the beginning of the next mapping event, the place-holder slots for tasks that have not begun execution are freed, which allows the execution of any newly arrived tasks of higher value. The tasks that were assigned placeholder slots during a mapping event will be present in the mappable set when the heuristic is called in subsequent mapping events, and if no other higher-value task becomes available, they may be executed as initially planned by the place-holder. In this way, the place-holder technique does not lose the benefits that reservations provide and adds flexibility for future mapping events.

In an oversubscribed environment, it may help to pick resource allocation choices that provide the most value, while using the least amount of resources. Thus, we designed a new resource management heuristic called Maximum Value-per-Total Resource (Maximum VPTR). The framework for this greedy heuristic is based on the Min-Min technique (e.g., [4], [9], [18]), but with a very different set of conditions. The Maximum VPTR heuristic selects the choice of resources based on maximizing "task value earned / total amount of resources allocated". We define the total resources for task $i$ as:

$$
\text { total resources }=\text { execution time duration } \times
$$

$[(\%$ of total number of system cores used $)+$ (\% of amount of total system RAM used)].

In general, each of these percentages can be weighted for a given system, depending on factors such as the relative cost of cores and memory or relative demand for cores versus memory among applications in typical workloads. For this study, we assumed an equal weighting. Our heuristic (shown in Algorithm 1) operates as follows.

For each mappable task $i$, independently, for each of its allowable resource configurations, we find the earliest time when that task $i$ has the needed number of cores and the needed amount of RAM available. Then, based on the completion time and the energy consumption, we calculate the VPTR for that configuration for task $i$. We select the task $i$ configuration that provides the best VPTR for task $i$ as shown in lines 1 to 3 of Algorithm 1. (If there is no configuration that results in a non-zero value, that task is dropped from the set of mappable tasks because it cannot contribute any value to the $\operatorname{VoS}$ measure.) Then, among all mappable tasks, we select the task that has the maximum VPTR, call this task $m$, and make that assignment of resources to task $m$, or if the allocation is for a future time, create a place-holder for task $m$. We then update the system state information based on this 
assignment or place-holder, and remove task $m$ from the list of mappable tasks. These steps of selecting and assigning a task are shown in lines 4 to 10 of Algorithm 1. This process is then repeated from the beginning until no more mappable tasks exist.

If there are multiple choices for the set of cores that have the earliest start time for a given task, we use a core selection procedure based on our prior work in [13]. Given the cores are homogeneous, a task's execution time will be the same irrespective of which cores are assigned. Because of this, finding the earliest possible completion time for a task (which maximizes the performance value) is equivalent to finding the task's earliest possible start time. When the earliest possible starting time for a task is found, it is possible that there will be multiple sets of cores that can be used. We use two criteria to attempt to choose a set to use. The first of these criteria is to pick cores that cause the smallest number of idle voids in the system (i.e., sections of time between the executions of two tasks). The second criterion is to compare the size of the idle voids into which the task would be inserted, and to choose the nodes with the smallest voids. Please see [13] for details. The motivation for these criteria is to reduce the overall fragmentation of the schedule to give future tasks a better chance of being backfilled.

For comparison, we considered the Simple heuristic, which assigns the mappable tasks in a random order. For each task, it chooses randomly one of its allowable resource configurations. The task is then assigned to the earliest possible time. If a task is unable to start executing immediately, then a permanent reservation is created for it on those resources instead. If there are multiple choices for the set of cores that have the earliest start time for a given task, Simple just uses the cores with the smallest "id" numbers rather than using the core selection procedure above. The task is then removed from the set of mappable tasks. This process, shown in Algorithm 2, is repeated until there are no more mappable tasks. We designed, implemented, evaluated, and compared three additional variations of this heuristic. The first, Simple w/ dropping, does not make assignments that would earn zero value. Simple $w$ / place-holders uses place-holders instead of permanent reservations. Finally, Simple $w /$ dropping and placeholders includes the concepts of place-holders and dropping.

\section{SIMULATION STUDY \\ 5.1 Simulation Setup}

To compare our heuristic techniques, we simulate twelve hours of task arrivals with the first two hours as warm-up for steady state. Statistics are collected starting from the third hour until the end. If a task begins execution but does not complete during that interval or finishes execution during the interval but starts its execution prior to the beginning of the interval, then the system will earn a portion of the task's value. We model ten different system environments. In five, the system memory is fixed at $256 \mathrm{~GB}$ and the number of cores is varied between 128 and 384. In the other five, the number of cores is fixed at 256 and system memory is varied from 128 to $384 \mathrm{~GB}$.

We simulate 48 different scenarios with a varying number of tasks, their types, and characteristics (i.e., the allowable resource configurations and associated execution times and energy needs). The number of tasks arriving for each simulation scenario is varied between 600 and 800 tasks, where each task belongs to one of 30 to 40 task types. The range used for the number of tasks was selected to ensure that the system is oversubscribed. The number
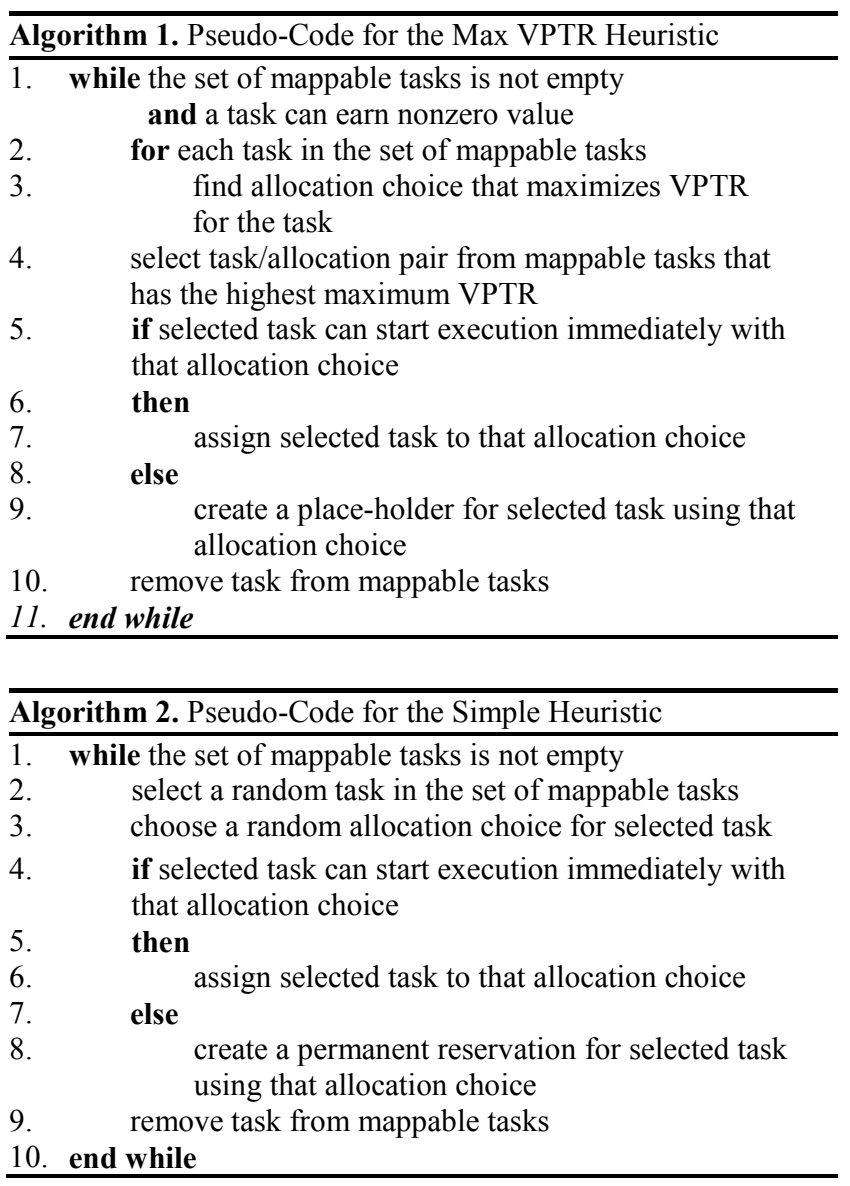

of tasks and types are determined by a uniform distribution and task inter-arrival follows a sinusoidal pattern.

Each task type has a number of allowable resource configurations specifying from 4 to 32 cores and from 6 to 40 GB of memory. We create a base case for each task type that defines its execution characteristics given a single core and one GB of memory. The system base case values for a scenario are sampled using Gaussian distributions with means of 150 minutes for execution time and 100 joules for energy consumption. The COV (coefficient of variation) of both distributions is 0.1 . For each task type, the base case values are generated with Gaussian distributions that use the system base case value as the mean and a COV of 0.2 . If a sampled value falls outside of $40 \%$ to $160 \%$ of the system base case value, then it is set to the minimum or maximum of this range, respectively. This method is based on the COV method used in [2]. The execution times of the allowable resource configurations are scaled from these base case values of that task type by using the Downey model for the speedup of parallel programs [6], with a uniformly sampled Downey sigma value between 4 and 10 . In addition, we scale the resulting execution time based on the amount of memory that is specified in the resource configuration. The energy consumption that corresponds to each resource configuration for a task type is linearly scaled using the number of cores and memory allocated.

Each task type has an importance between 1 and 10 that is sampled from a Gaussian distribution with a mean that is correlated directly with the task type's base case single core execution time and a COV of 0.2 . For VoS, the performance value function and the energy value function are weighted equally (i.e., 
$\left.w_{p}=w_{e}\right)$. Each value function has a $v_{\max }\left(t_{j}\right)$ value of 1 and a $v_{\min }\left(t_{j}\right)$ value distributed uniformly over the range 0 to 1 . The hard thresholds for the performance and energy value functions are created using Gaussian distributions with a COV of 0.2 and means equal to the highest terms (in execution time and energy consumption). If the generated value for the hard threshold is less than the highest term, the distribution is resampled. The soft thresholds have a COV of 0.2 and a mean equal to the lowest term plus 0.05 times the difference between the lowest and highest terms. A soft threshold is resampled if it is greater than the corresponding hard threshold.

\subsection{Simulation Results}

The results in this section were averaged over 48 simulation scenarios and are shown with $95 \%$ confidence intervals. To better compare the performance of heuristics, we plot their total value earned as a percentage of the maximum system value possible for that scenario. We define the maximum system value as the value earned by the system if all tasks started execution when they arrived in the system with the execution time of their fastest allocation choice and the energy consumption of their allocation choice with the lowest energy usage (this is an upper bound). These values usually come from different allocation choices, but they are used to ensure that an upper bound is calculated in all cases.

Figure 2 and 3 show the percentage of maximum system value earned by each of the heuristics. Figure 2 shows the system memory fixed at $256 \mathrm{~GB}$ with the number of cores varied between 128 and 384. Figure 3 shows the system with number of cores fixed at 256 and system memory varied from 128 to $384 \mathrm{~GB}$. The Simple heuristic has very poor performance in all environments, but its performance improves when dropping or place-holders are used with it.

Our value-based heuristic utilizes additional information to choose tasks and resource configurations that may result in better system performance. Figure 2 and 3 show that the value-based heuristic is able to earn higher value than the Simple heuristic. When the system has a limited number of cores (i.e., 128) or a limited amount of memory (i.e., $128 \mathrm{~GB}$ ) available, the heuristic is able to perform well because it takes the execution time of and number of cores or amount of memory allocated to a task into account to choose allocation choices that utilize the limited resources (cores or memory) efficiently.

These simulations show that, using place-holders and dropping allows the Simple and Max VPTR heuristics to perform well in a variety of environments.. The Max VPTR heuristic is able to outperform the Simple heuristic using place-holders and dropping in all of the environments that were simulated. The advantage of the Max VPTR heuristic is that it incorporates the resources needed to execute tasks, and in all our studies of oversubscribed systems at least one of the number of nodes or the amount of memory is a limitation on system performance.

\section{EXPERIMENTAL RESULTS}

\subsection{Data-Driven Model}

We design a power model to track energy consumption of each task and to calculate the value of energy earned upon completion since the energy consumed by individual VMs cannot be measured using any device. Hence, we use a data-driven approach to model the power consumption of HPC tasks. Initially, we collect a wide range of parameters associated with CPU and

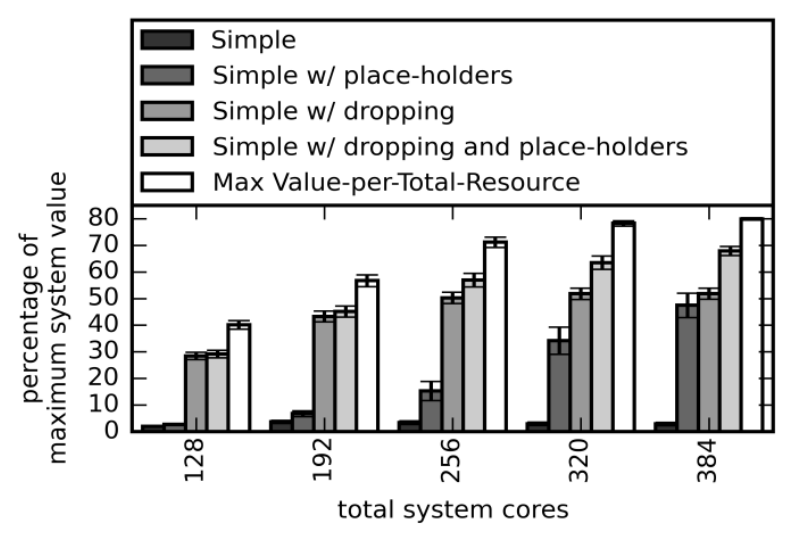

Figure 2. The percentage of maximum system value earned by the heuristics in environments where the number of cores in the system is varied from 128 to 384 and the amount of memory is fixed at $256 \mathrm{~GB}$.

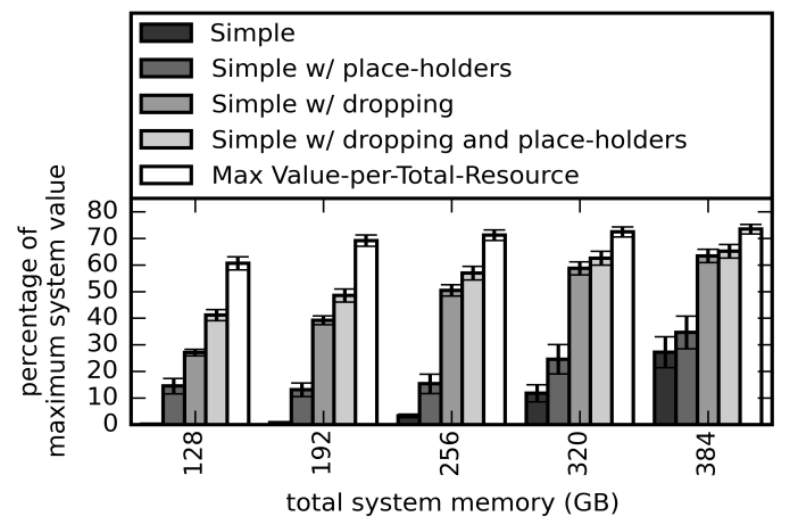

Figure 3. The percentage of maximum system value earned by the heuristics in environments where the amount of memory in the system is varied from 128 to 384 GB and the number of cores is fixed at 256 .

memory operations as these operations are the primary contributors to the overall VM power consumption as shown by [3] and [11]. We use the Perf tool for tracking hardware performance counters for VMs launched [21]. To identify the set of performance counters that are highly correlated with power consumption, we generate two sets of micro benchmarks. The first set involves integer and floating point arithmetic operations such as summation, multiplication, and division for identifying the individual CPU parameters. During the arithmetic operations, the memory operations are kept to a minimum. The second set involves memory reads and writes in terms of copy operations over various sizes of memory blocks. Next we use our parameter selection algorithm [22] to identify the most relevant ones for characterizing the power consumption. Table 2 lists those parameters identified for this work.

Next, we model the power consumption of the individual VMs based on the selected performance parameters. We first discretize $\mathrm{VM}$ power values into steps of 5 Watts in the range of 0 to 100 Watts, which we refer to as Power $_{\text {level }}$. Discretization reduces the computation complexity of the training process, with minor error in power estimates. Based on the data obtained from the micro benchmarks, we express each power level as a function of performance parameters using the JRip (a classifier implementation 
Table 1. Selected value function parameters

\begin{tabular}{|l|l|}
\hline $\begin{array}{l}\text { CPU only } \\
\text { filtered } \\
\text { parameter set }\end{array}$ & $\begin{array}{l}\text { cycles, L1-dcache-loads, L1-dcache-stores, L1- } \\
\text { dcache-store-misses, L1-icache-loads, L1-icache- } \\
\text { load-misses, dTLB-loads, dTLB-stores, dTLB-store- } \\
\text { misses, branch-loads, Power }\end{array}$ \\
\hline $\begin{array}{l}\text { Memory } \\
\text { only filtered } \\
\text { parameter set }\end{array}$ & $\begin{array}{l}\text { cache-misses, cpu-clock(msec), task-clock(msec), L1- } \\
\text { dcache-stores, L1-dcache-prefetches, L1-icache- } \\
\text { loads, LLC-load-misses, LLC-stores, LLC-store- } \\
\text { misses, dTLB-stores, branch-loads, Power }\end{array}$ \\
\hline
\end{tabular}

based on the RIPPER algorithm we use for power level correlation with the selected parameters). One such rule is shown in Figure 4.

In our earlier work ([7], [8], [24]) on dynamic resource allocation for the individual VMs of the cloud computing systems, we used the same method for characterizing the workload behavior and its resource requirements. We train the model based on micro benchmarks and evaluate its accuracy based on NAS-NPB [20] benchmarks. Our model predicts power consumption of the VMs running these benchmarks with $90 \%$ accuracy. We discuss the nature of these benchmarks in Subsection 6.3.

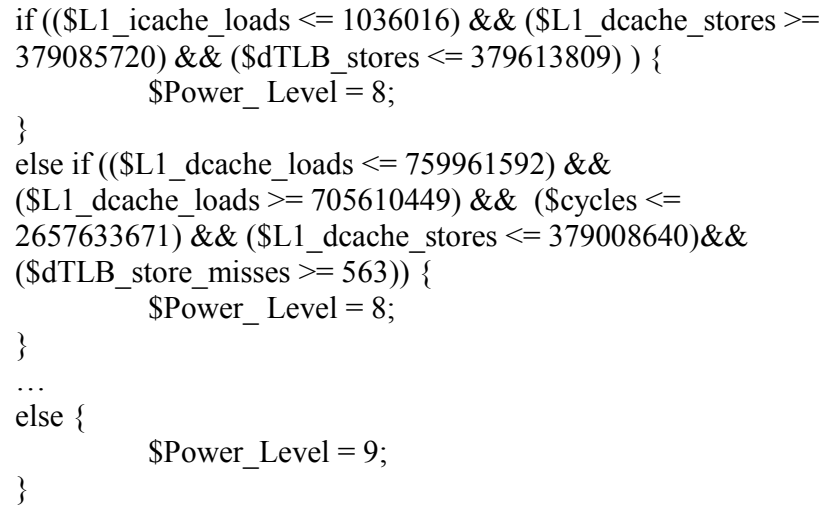

Figure 4. Sample rule for the power level 8 (40 Watts)

\subsection{Experimental Setup}

System and Benchmarks: We use an IBM HS22 blade server with four nodes. Each node consists of two Intel Xeon X5650 6core processors $(2.67 \mathrm{GHz})$ and $24 \mathrm{~GB}$ RAM. We use Kernelbased Virtual Machine (KVM) for hypervisor and Perf tool for profiling. We record the power consumption using IBM Advanced Management Module [10].

A VM is assigned to specific cores on a single node in our blade server, and a VM cannot be split across multiple nodes, but a single node can have multiple VMs. Each task is assigned to a specific VM and cannot be split across VMs. Resource allocation for a VM on a particular node is limited by the physical resources available at that point of time on that node and VMs do not share hardware resources.

Similar to the simulation, the cluster is considered as an oversubscribed system with a set of independent tasks arriving dynamically. To demonstrate this scenario, we use the following four kernels from the MPI based NAS-NPB [20] benchmarks:

- FT - discrete 3D fast Fourier Transform

- IS - Integer Sort, random memory access

- EP - Embarrassingly Parallel

- LU - Lower-Upper Gauss-Seidel solver

NAS-NPB allows adjusting the problem size, ranging from class A to F (largest). For example, for FT kernel, class A processes a

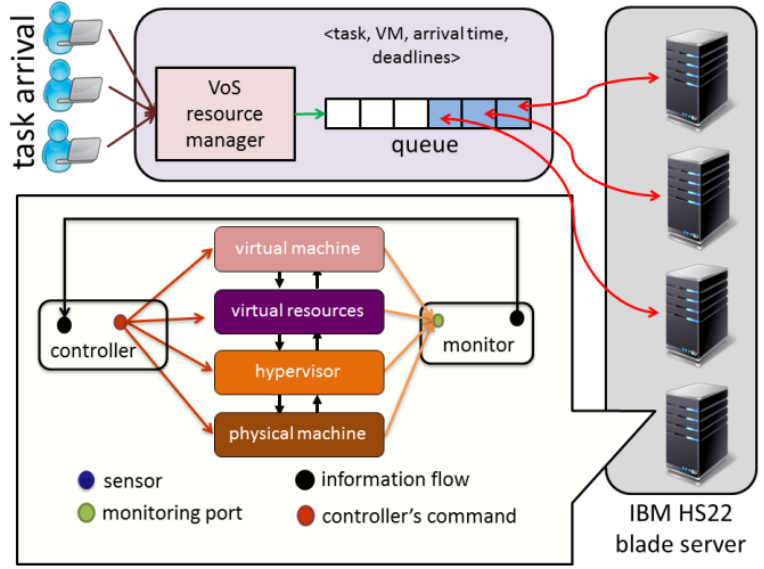

Figure 5. The overall scheduling architecture which consists of the resource manager, queue, and the HPC system.

256x256x128 matrix whereas class F processes 4096x2048x2048. For the experiments, we use class $\mathrm{C}$ for data size.

Workload Generation: We generate a workload trace for simulating the task arrival and store in a queue monitored by the scheduler. Each task is associated with a task type, its arrival time, and its soft and hard completion time thresholds. The difference between arrival times of two consecutive tasks is sampled using a Gaussian distribution such that the system is oversubscribed. Task type is chosen using a uniform distribution. Soft and hard completion thresholds are calculated using the same methodology as described under the simulation environment. Each of the experiments was executed for a duration of one, two, and four hours, which we refer to as the monitored duration. During generation of task arrival trace, we ensure that no task arrives after the monitored duration. At the end of each monitored duration, using the $\operatorname{VoS}$ equation, the value earned by the scheduled workload tasks is calculated for the system.

Figure 5 illustrates the scheduler design which consists of the resource manager, a queue, and the HPC system. The resource manager first determines the VM configuration requirements of the selected task based on a given heuristic and then assign that task to a node with sufficient resources. Each heuristic under investigation uses the Estimated Time to Compute (ETC) matrix and the Estimated Energy Consumption (EEC) matrix to determine VM allocations and scheduling.

Depending on the resource manager, a local controller on physical node creates the required VM, starts the Perf tool, and executes its assigned task. To avoid VM generation overhead, we have a set of VMs ready to be launched with the required executables. When the task is completed, monitored performance counter values and with task execution time are used to calculate energy consumption using the power model introduced in Subsection 6.1. After the termination of the VM, the local controller updates the task queue indicating the task has been completed. We calculate the value earned by the system after completing each task using Equation 2. The earned values for all of the tasks are accumulated into $\operatorname{VoS}$ at the end of the experiment. We use the VoS metric for comparing the performance of the heuristics for each workload type (trace).

\subsection{Experimental Results}

In this subsection, we evaluate the Simple with dropping and place holders (Simple w/d\&p) and VPTR heuristics over three 


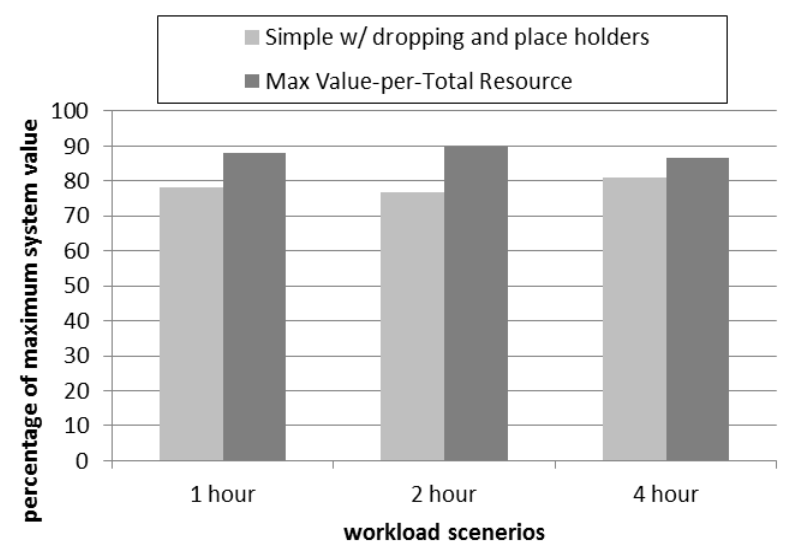

Figure 6. The total value earned for different workload scenarios.

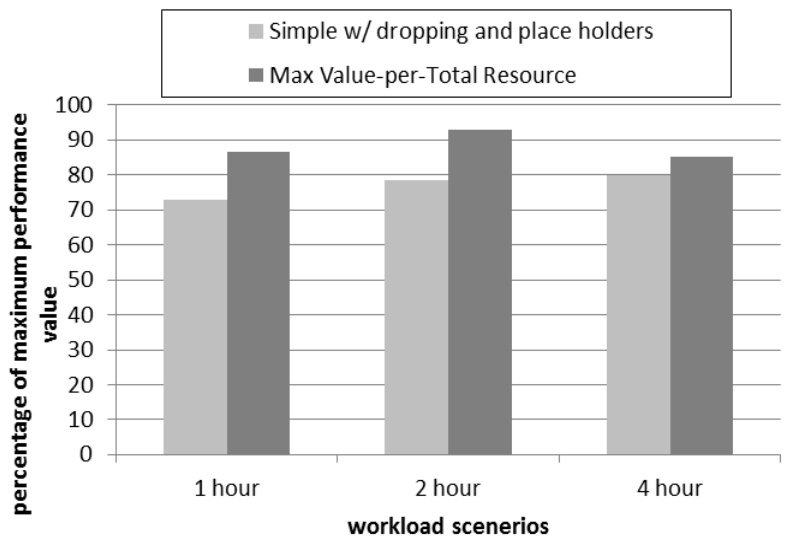

Figure 7. Total performance value earned for different workload scenarios.

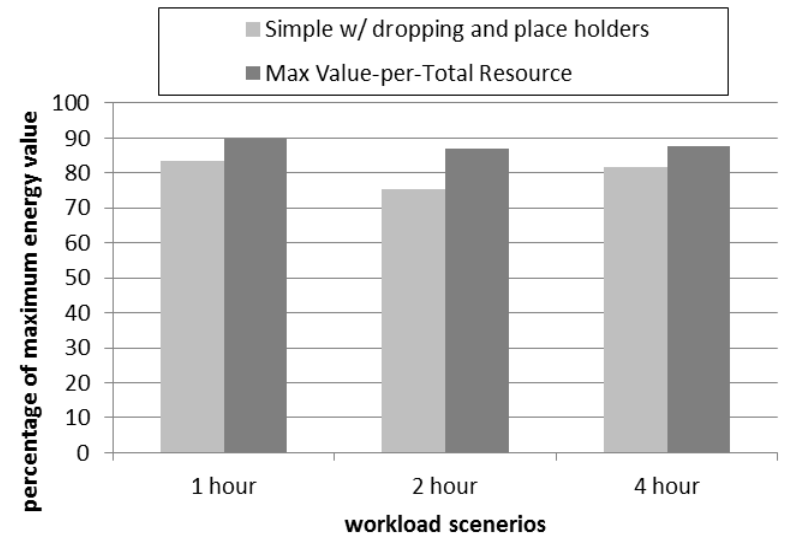

Figure 8. Total energy value earned for different workload scenarios.

types of workload scenarios. In Figure 6, we show the percentage of the total values earned by both approaches. VPTR consistently outperforms the Simple w/d\&p heuristic in these experiments, confirming the simulation results shown in Figures 2 and 3.

In Figures 7 and 8, we show separately the total performance and energy values earned by each heuristic, respectively. Based on Figure 7, which shows the total performance value earned by each heuristic, we conclude that the VPTR heuristic is making better resource allocation decisions than Simple w/d\&p in these experiments executed on real hardware with standard benchmarks, for all three workload scenarios. In our experiments, we have not observed a significant difference between the total energy consumptions of the two heuristics, which indicates that the resource usage of the blade system is similar for both of the heuristics. However, when we consider Figure 8, it can be seen that the energy value earned by VPTR versus Simple w/d\&p shows a clear benefit of VPTR. This is because the VPTR heuristic makes informed decision in resource allocation by taking energy and execution time into account whereas decisions taken by the Simple w/d\&p is random.

\section{RELATED WORK}

There is a large body of work on resource management strategies for HPC systems targeting various metrics such as system resource utilization, task execution time, and total power/energy consumption. Most of the resource management algorithms utilize backfilling ([1], [5], [13], [15], [19]) for resource utilization and execution time. Our work differs from these other studies in that the VoS metric allows us to target two objectives (performance and energy) concurrently by flexibly weighting their relative significance for each task during their scheduling. Additionally, we include the relative importance among tasks, and evaluate the total value earned by the system for a given workload in a given interval of time. Furthermore, the system models used by the earlier studies do not consider VMs.

For example, the value function introduced in [13] evaluates the value for completed tasks in an oversubscribed system. The value function takes only task completion time into account. Also, its system model does not consider using VMs to allocate cores and memory. In [13], heterogeneous cores are explicitly allocated. The energy aware utility based heuristics studied in ([12], [17]) treat energy as a constraint instead of a component of the overall VoS. Utility maximization is also targeted in [25] under an energy constraint during oversubscription by prioritizing the scheduling of tasks with the highest magnitude of utility per unit energy at a particular instant of time. In this method, there is a chance for higher value tasks to be mapped to a node with fewer resources (core/memory), which would lead to increasing total execution time and also an increase in the total energy consumption.

A profit- and penalty-aware scheduling algorithm introduced in [16] associates a task with two Time Utility Functions (TUFs): a profit TUF and penalty TUF. The profit TUF is defined as a utility earned by a system based on timely completion of a task. Penalty TUF is defined in terms of utility lost by dropping the task or missing its completion deadline. If two tasks have the same profit TUF, but different penalty TUF, then their approach will favor scheduling the task with the lower penalty TUF. In our approach, tasks are not penalized for achieving their soft thresholds (minimum energy and execution time requirement); therefore we treat both tasks equally for scheduling decisions. We feel this promotes fair allocation of resources for tasks with equal value. We also schedule a task only if it can earn any value, and once scheduled a task cannot not be dropped.

\section{CONCLUSIONS}

In this paper, we introduce a new metric, Value of Service (VoS), that measures the total value earned by completing the tasks of a given workload during a given time interval. We model worth of completing a task before its deadline by a performance value function and worth of completing a task within a certain range of energy consumption by an energy value function. We propose a 
weighting scheme for these two components that can be adjusted based on the system environment. We include an importance factor to specify the relative significance among tasks. We combine all these parameters to form a task value measure, and sum them over the tasks completed during a given interval of time to create the overall system performance metric $\operatorname{VoS}$. VoS is novel as it takes task completion time, energy consumption, and importance of a task into account. We introduce new resource management heuristics based on the VoS metric, and show that the heuristics we designed are an effective tool for resource management based on simulation and experimental based studies.

\section{ACKNOWLEDGMENTS}

This research was supported by NSF grant CCF-1302693. This research used the CSU ISTeC Cray System supported by NSF Grant CNS-0923386.

\section{REFERENCES}

[1] Alem, A.W.M. and Feitelson, D.G. Utilization, predictability, workloads, and user runtime estimates in scheduling the IBM SP2 with backfilling. IEEE Trans. Parallel and Distributed Systems, 12(6) 529-543, June 2001

[2] Ali, S., Siegel, H.J., Maheswaran, M., Hensgen, D. and Ali, S. Representing task and machine heterogeneities for heterogeneous computing systems. Tamkang J. Science and Engineering, 3(3) 195-207, Nov. 2000, invited.

[3] Bohra, A.E.H. and Chaudhary, V., VMeter: Power modelling for virtualized clouds. Workshop on High-Performance, Power-Aware Computing, IEEE Int'1 Parallel and Distributed Processing Symp. Workshops (IPDPSW), 1-8, Apr. 2010

[4] Braun, T., Siegel, H.J., Beck, N., Bölöni, L.L., Maheswaran, M., Reuther, A.I., Robertson, J.P., Theys, M.D., Yao, B., Hensgen, D. and Freund, R.F. A comparison of eleven static heuristics for mapping a class of independent tasks onto heterogeneous distributed computing systems. J. Parallel and Distributed Computing, 61(6), 810-837, June 2001.

[5] Davis, R.I. and Burns, A. A survey of hard real-time scheduling for multiprocessor systems. ACM Computing Surveys, 43(4), Oct. 2011.

[6] Downey, A.B. Model for speedup of parallel programs. Tech. Rep. UCB/CSD-97-933. University of California, Berkeley, 1997.

[7] Fargo, F., Tunc, C., Al-Nashif, Y., Akoglu, A. and Hariri, S., Autonomic Workload and Resources Management of Cloud Computing Services. IEEE Int'l Conf. Cloud and Autonomic Computing (ICCAC), 101-110, Sep. 2014.

[8] Fargo, F., Tunc, C., Al-Nashif, Y., and Hariri, S. Autonomic performance-per-watt management (APM) of cloud resources and services. ACM Cloud and Autonomic Computing Conf., Aug. 2013.

[9] Ibarra, O.H. and Kim, C.E. Heuristic algorithms for scheduling independent tasks on non-identical processors. $J$. ACM, 24(2) 280-289, Apr. 1977.

[10] IBM BladeCenter Advanced Management Module (User's Guide), May 2013. //publib.boulder.ibm.com/infocenter/bladectr/documentation/ topic/com.ibm.bladecenter.advmgtmod.doc/kplbb_pdf.pdf.

[11] Kansal, A., Zhao, F., Liu, J., Kothari, N. and Bhattacharya, A.A. Virtual machine power metering and provisioning. 1st ACM Symp. on Cloud Computing, 39-50, June 2010.
[12] Khemka, B., Friese, R., Pasricha, S., Maciejewski, A.A., Siegel, H.J., Koenig, G.A, Powers, S., Hilton, M., Rambharos, R., and Poole, S. Utility maximizing dynamic resource management in an oversubscribed energyconstrained heterogeneous computing system. Sustainable Computing: Informatics and Systems, 5, 14-30, Mar. 2015.

[13] Khemka, B., Machovec, D., Blandin, C., Siegel, H.J., Hariri, S., Louri, A., Tunc, C., Fargo, F. and Maciejewski, A.A. Resource management in heterogeneous parallel computing environments with soft and hard deadlines. 11th Metaheuristics Int'l Conf. ( MIC), June 2015.

[14] Khokhar, A., Prasanna, V.K., Shaaban, M.E., and Wang, C. Heterogeneous computing: Challenges and opportunities. IEEE Computer, 26 (6), 18-27. June 1993.

[15] Lifka, D.A. The ANL/IBM SP scheduling systems. Workshop on Job Scheduling Strategies for Parallel Processing, 295-303, Apr. 1995.

[16] Liu, S., Quan, G., and Ren, S. On-line scheduling of realtime services for cloud computing. 6th World Congress on Services, 459- 464, 2010.

[17] Machovec, D., Khemka, B., Pasricha, S., Maciejewski, A.A., Siegel, H.J., Koenig, G.A., Wright, M., Hilton, M., Rambharos, R., and Imam, N. Dynamic resource management for parallel tasks in an oversubscribed energyconstrained heterogeneous environment, $25^{\text {th }}$ Heterogeneity in Computing Workshop (HCW 2016), 2016 Int'1 Parallel and Distributed Processing Symp. Workshops (IPDPSW 2016), May 2016.

[18] Maheswaran, M., Ali, S., Siegel, H.J., Hensgen, D. and Freund, R.F. Dynamic mapping of a class of independent tasks onto heterogeneous computing systems. J. Parallel and Distributed Computing, 59(2) 107-131, Nov. 1999.

[19] Mishra, A., Mishra, S., and Kushwaha, D.S. An improved backfilling algorithm: SJF-B. Int'l J. Recent Trends in Engineering \& Technology, 5(1) 78-81, Mar. 2011,

[20] NAS Parallel Benchmarks (NAS-NPB). //www.nas.nasa.gov/publications/npb.html

[21] Perf: Linux Profiling with performance counters, 2016. //perf.wiki.kernel.org/index.php/Main_Page

[22] Qu, G., Hariri, S, and Yousif, M. A new dependency and correlation analysis for features. IEEE Trans. Knowledge and Data Engineering, 17(9) 1199-1207, Aug 2005.

[23] Top Ten Exascale Resource Challenges. 2014. DOE ASCAC Subcommittee Report, Feb. 2014. //science.energy.gov/ /media/ascr/ascac/pdf/meetings/20140 210/Top10reportFEB14.pdf

[24] Tunc, C. Autonomic Cloud Resource Management. The University of Arizona, 2015, PhD Thesis.

[25] Wu, H., Ravindran, B., and Jensen, E.D. Energy-efficient, utility accrual real-time scheduling under the unimodal arbitrary arrival model. IEEE/ACM Design, Automation and Test in Europe, 474-479, 2005.

[26] Xu, D., Nahrstedt, K., and Wichadakul, D. QoS and contention-aware multi-resource reservation. Cluster Computing, 4(2), 95-107, Apr. 2001. 